\title{
Sequential Ultrafiltration-Catalysis Membrane for Excellent Removal of Multiple Pollutants in Water
}

Jia Xie,${ }^{\dagger}$ Zhipeng Liao, ${ }^{\dagger}$ Ming Zhang, ${ }^{\dagger}$ Linhan Ni, ${ }^{\dagger}$ Junwen Qi, ${ }^{\dagger}$ Chaohai Wang, ${ }^{\dagger}$ Xiuyun Sun, ${ }^{\dagger}$ Lianjun Wang, ${ }^{\dagger}$ Shaobin Wang ${ }^{\ddagger}$, Jiansheng $\mathrm{Li} *, \dagger$

†Key Laboratory of New Membrane Materials, Ministry of Industry and Information Technology, School of Environmental and Biological Engineering, Nanjing University of Science and Technology, Nanjing 210094, China

${ }^{\ddagger}$ School of Chemical Engineering, The University of Adelaide, Adelaide, SA 5005, Australia

The Supporting Information included:

21 Pages

16 Figures

3 Texts 


\section{Text S1.}

A modified kinetic model $\left(k^{\prime}\right)$ based on degradation rate was used to evaluate the activation of PMS. The formula was described in the following equation. ${ }^{1}$

$$
k^{\prime}=\frac{1}{m_{\text {cat }}} \frac{d C}{d t}
$$

Where, $n$ is the amount of organic pollutants $(\mu \mathrm{mol}), t(\mathrm{~s})$ is reaction time and $m_{\text {cat }}$ is the mass of catalysts $(\mathrm{g})$. For a filtration oxidation process through a membrane, the firstorder kinetics was described by eq. $2 .^{2}$

$$
-\frac{d C_{x}}{x}=-\frac{d C_{x}}{t} \frac{d t}{d x}=\frac{k C_{X}}{v}
$$

Where, $C_{x}$ is the concentration of BPA at a distance $\mathrm{x}$ into the membrane, $k\left(\mathrm{~min}^{-1}\right)$ is the first-order rate constant, and $v$ is the linear velocity of the feed solution in the membrane. 


\section{Text S2.}

The filtration performances, catalytic self-cleaning and antifouling properties of membranes were investigated by a dead-end filtration system with stirred cells (Model 8050, Merck Millipore Inc., America), and the effective filtration area of the membrane is $13.4 \mathrm{~cm}^{2}$.

The water flux and solute rejection of the membranes were tested with DI water and $1 \mathrm{~g}$ $\mathrm{L}^{-1} \mathrm{BSA}$ at 1 bar, respectively. Pure water flux $(J)$ and the retention rate of BSA $(R)$ were calculated from

$$
\begin{gathered}
J=\frac{V}{A t} \\
R(\%)=\frac{C_{0}-C_{p}}{C_{0}} \times 100
\end{gathered}
$$

where $\mathrm{V}$ is the permeate volume, $A$ is the active membrane area, $\mathrm{t}$ is the interval time, $C_{p}$ is the BSA concentration in permeate solution, and $C_{0}$ is the initial BSA concentration in the feed solution. The concentrations of the BSA and PEGs were determined from absorbance at $280 \mathrm{~nm}$ with an UV-vis spectrophotometer (Lambda 25, PerkinElmer Inc., America).

Membrane porosity $(\phi)$ was calculated from the following equation:

$$
\phi(\%)=\left(\frac{M_{w}-M_{d}}{A d \rho}\right) \times 100
$$

where $M_{w}$ and $M_{d}$ are the weights of the wetted and dry membrane, respectively, $A$ is the membrane area, $d$ is the average membrane thickness, and $\rho$ is the density of water. 


\section{Text S3.}

HA ( $5 \mathrm{mg} \mathrm{L}^{-1}$ ) was employed to evaluate catalytic self-cleaning and antifouling properties of SUCM. The recovered water flux of fouled membrane was measured by hydraulic cleaning (immersing membrane in DI water) and backwashing with $0.1 \mathrm{~g} \mathrm{~L}^{-1}$ PMS solution.

The total fouling ratio $\left(R_{t}\right)$ was calculated using:

$$
R_{t}(\%)=\frac{J_{w}-J_{p}}{J_{w}} \times 100
$$

where $J_{w}$ is the pure water flux prior to fouling and $J_{p}$ is the water flux in the presence of HA foulant in the feed solution.

The self-cleaning performance of the UFM and SUCM was evaluated by calculating the water flux recovery ratios $\left(F_{r}\right)$ according the following equation:

$$
F_{r}(\%)=\frac{J_{c}}{J_{w}} \times 100
$$

where $J_{c}$ is the pure water flux after membrane cleaning. 


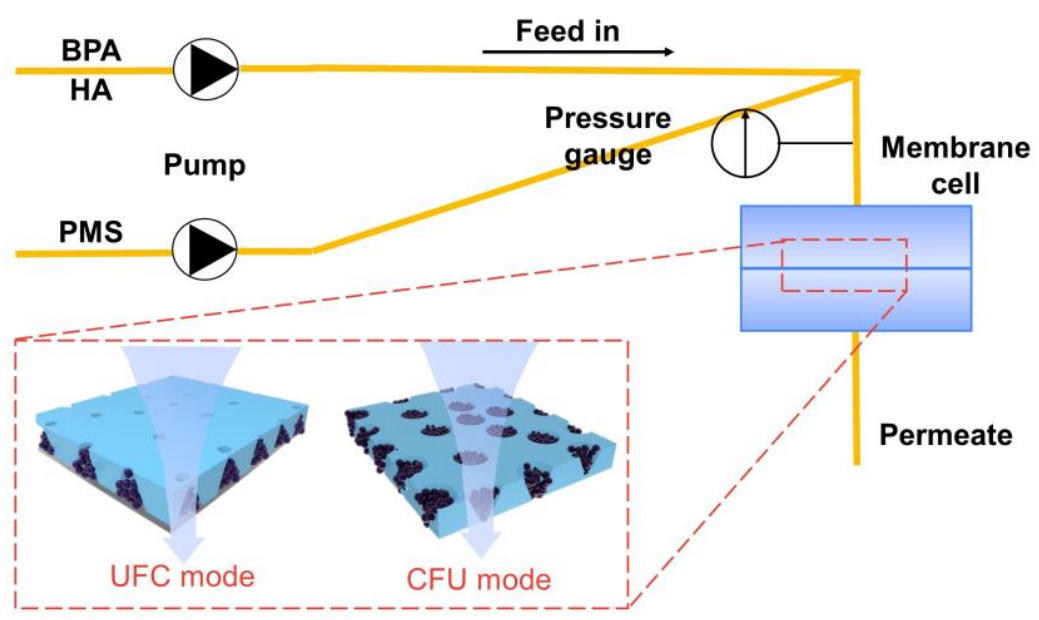

Figure S1. Scheme of membrane filtration operations. 


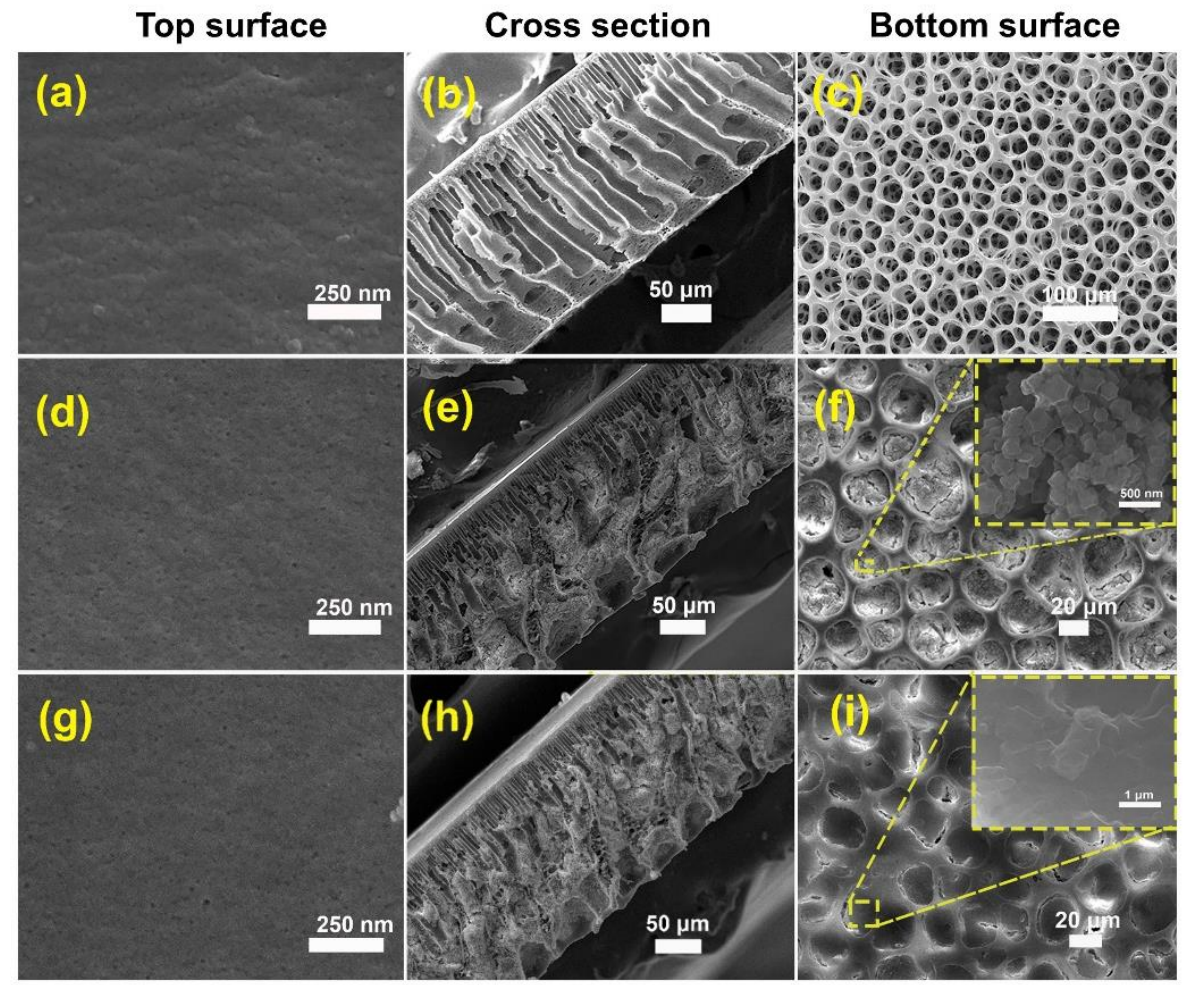

Figure S2. SEM images of top surface, cross section, and bottom surface of UFM (a-c), unsealed SUCM $(d-f)$, and SUCM $(g-i)$. 


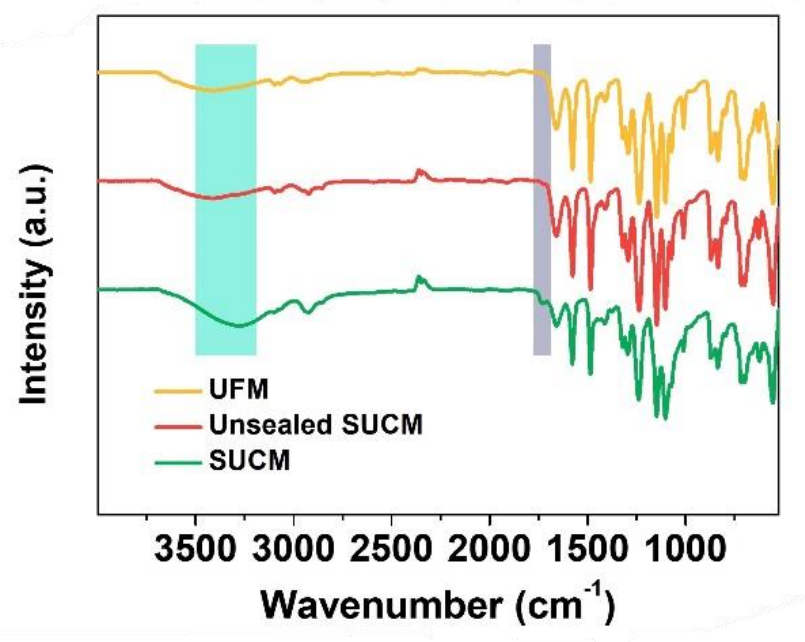

Figure S3. FTIR spectra of UFM, unsealed SUCM and SUCM.

To identify the sealing layer of PVA, FT-IR analysis of the membranes was investigated (Figure S4a). Vibration bands around $3270 \mathrm{~cm}^{-1}$ and $1725 \mathrm{~cm}^{-1}$ were observed, which were assigned to the stretching vibration of $\mathrm{O}-\mathrm{H}$ and $\mathrm{C}=\mathrm{O}$, respectively. The hydroxyl groups and ketone were ascribed to the polycondensation of PVA. ${ }^{3}$ 


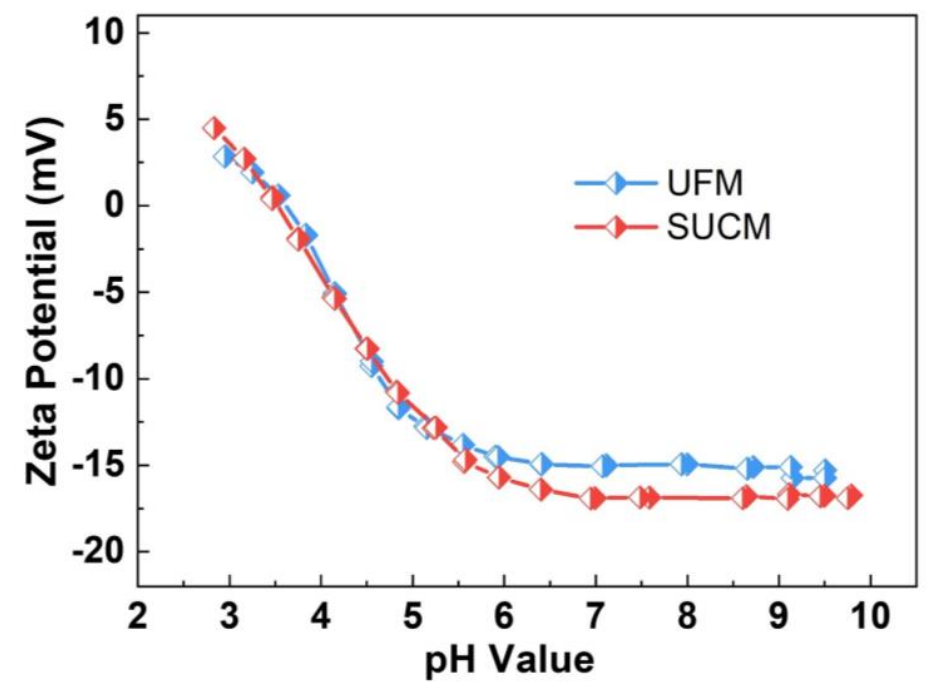

Figure S4. Zeta potentials of the membranes. 


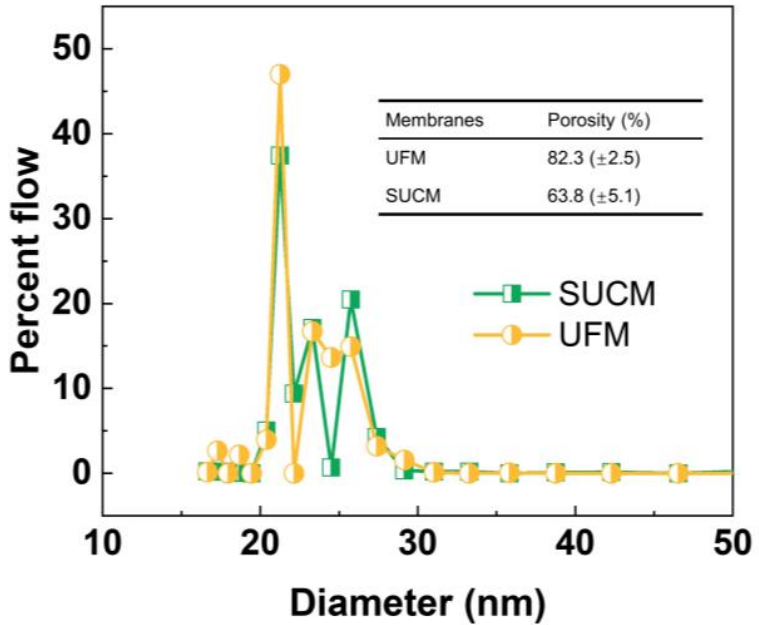

Figure S5. Pore size distribution of UFM and SUCM. The inset of Table shows the porosity of membranes. 


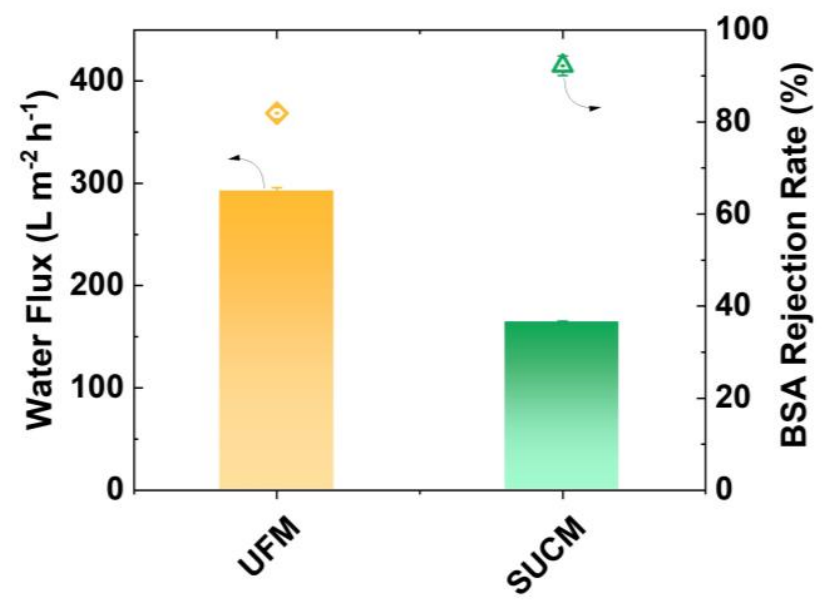

Figure S6. Filtration performance of the membranes. 


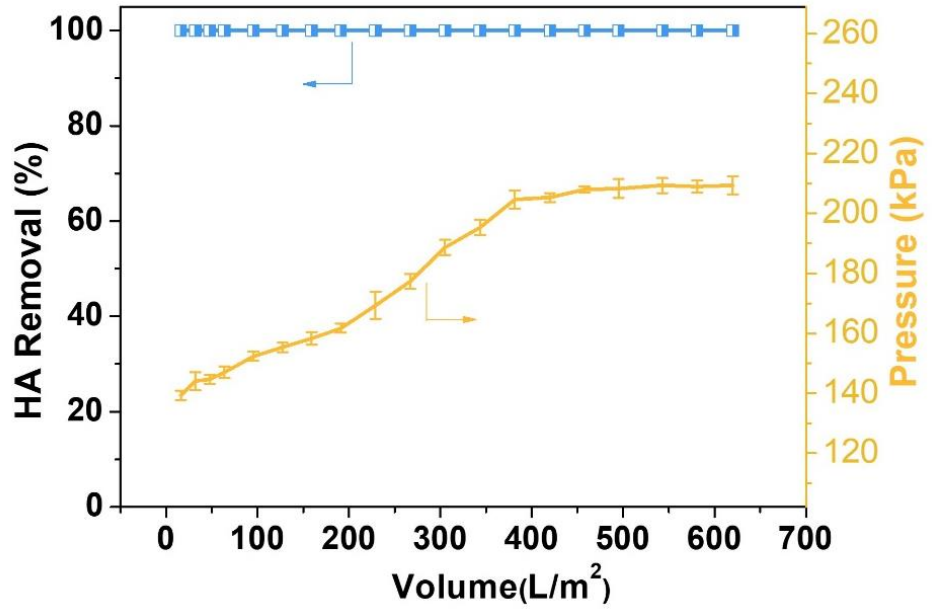

Figure S7. HA rejection and pressure of UFM (water flux $=229 \mathrm{~L} \mathrm{~m}^{-2} \mathrm{~h}^{-1}$ ). 


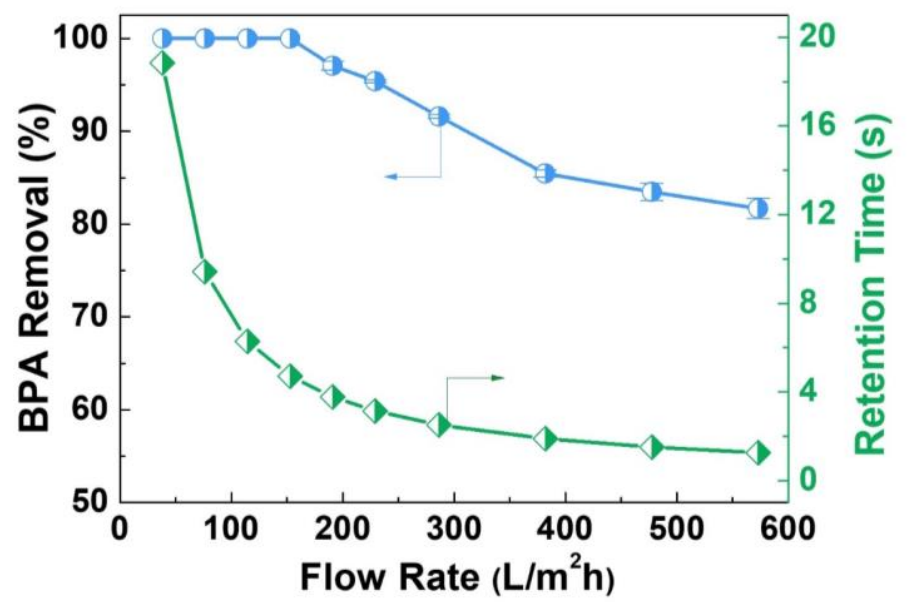

Figure S8. Effect of permeate flux on BPA degradation and the corresponding residence time. 


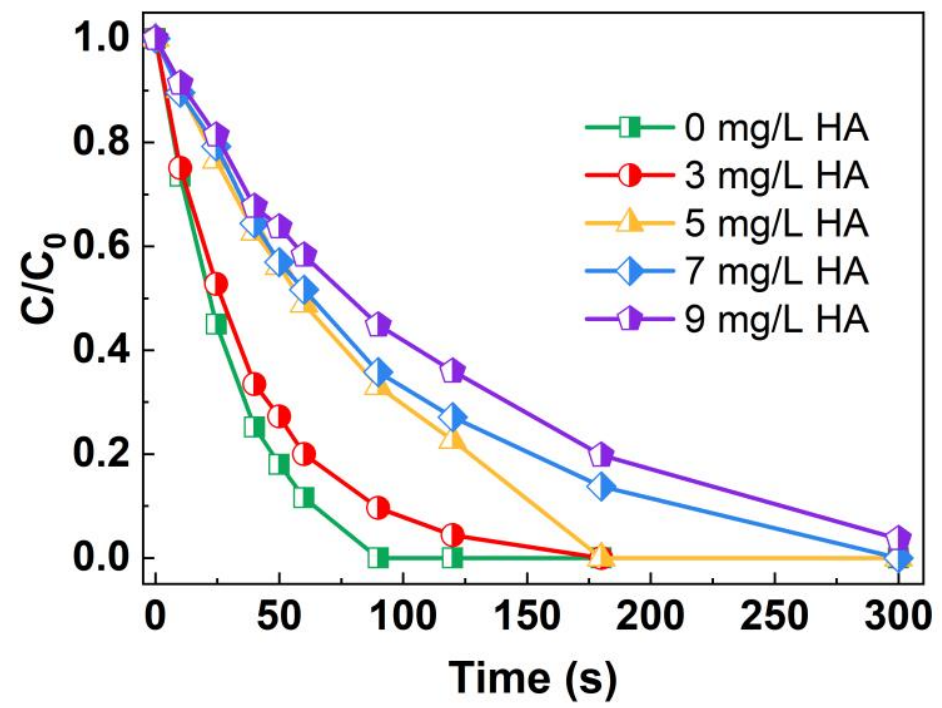

Figure S9. Effect of HA concentration BPA removal efficiency. Conditions: $[\mathrm{PMS}]_{0}=0.1 \mathrm{~g} \mathrm{~L}^{-1},[\mathrm{BPA}]_{0}=1$ $\mathrm{mg} \mathrm{L}^{-1}$, [cat $]_{0}=16 \mathrm{mg} \mathrm{L}^{-1}$, initial $\mathrm{pH}=7$. 


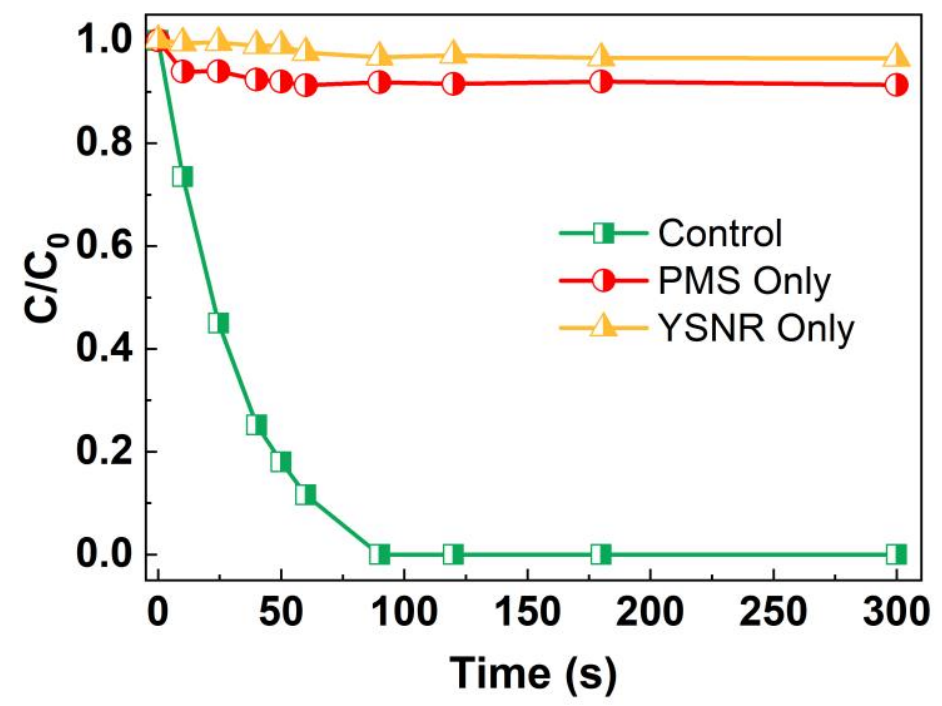

Figure S10. BPA removal efficiency in different reaction systems. Conditions: $[\mathrm{PMS}]_{0}=0.1 \mathrm{~g} \mathrm{~L}^{-1},[\mathrm{BPA}]_{0}=$ $1 \mathrm{mg} \mathrm{L}^{-1}$, [cat $]_{0}=16 \mathrm{mg} \mathrm{L}^{-1}$, initial $\mathrm{pH}=7$. 
(a)

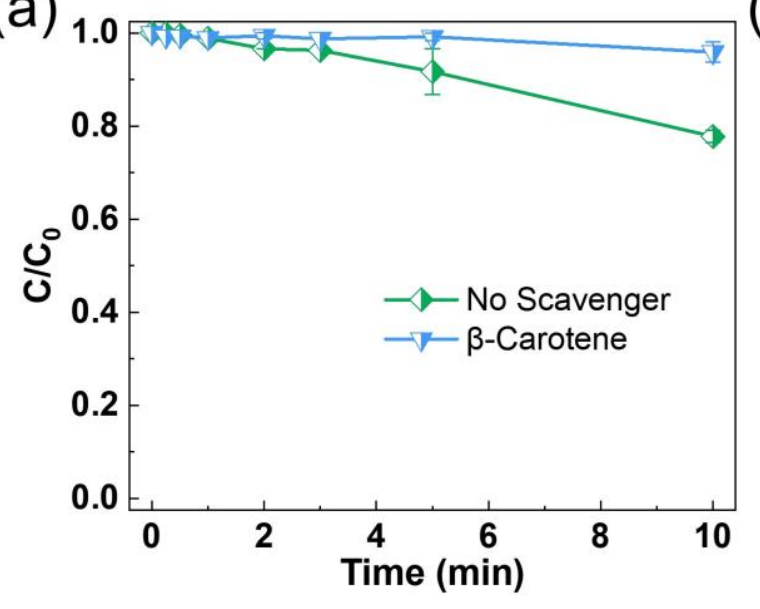

(b)

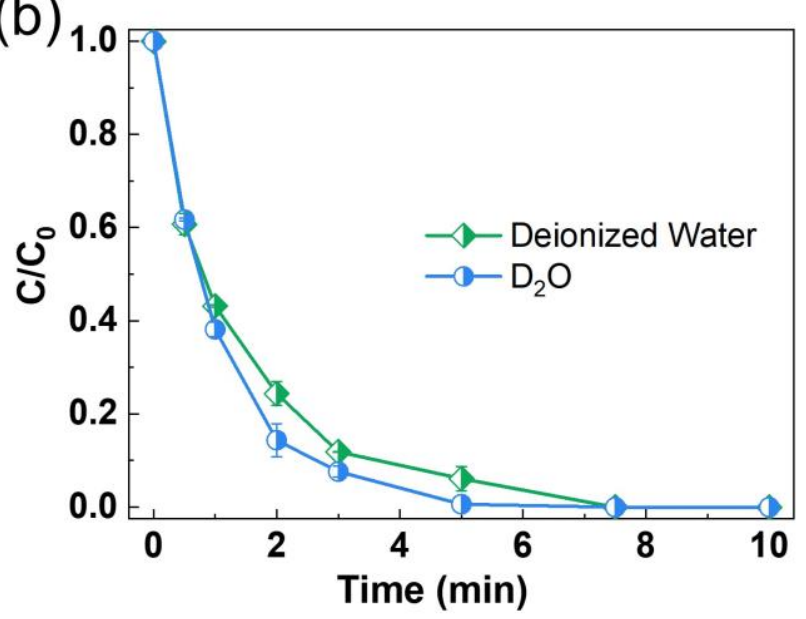

Figure S11. Effect of $\beta$-carotene (a) and $\mathrm{D}_{2} \mathrm{O}$ (b) on BPA removal efficiency. The effect of $\beta$-carotene was performed in $50 \mathrm{~mL}$ solution containing $47 \mathrm{~mL}$ acetone for dissolving $\beta$-carotene and $3 \mathrm{~mL}$ deionized water for dissolving PMS. 


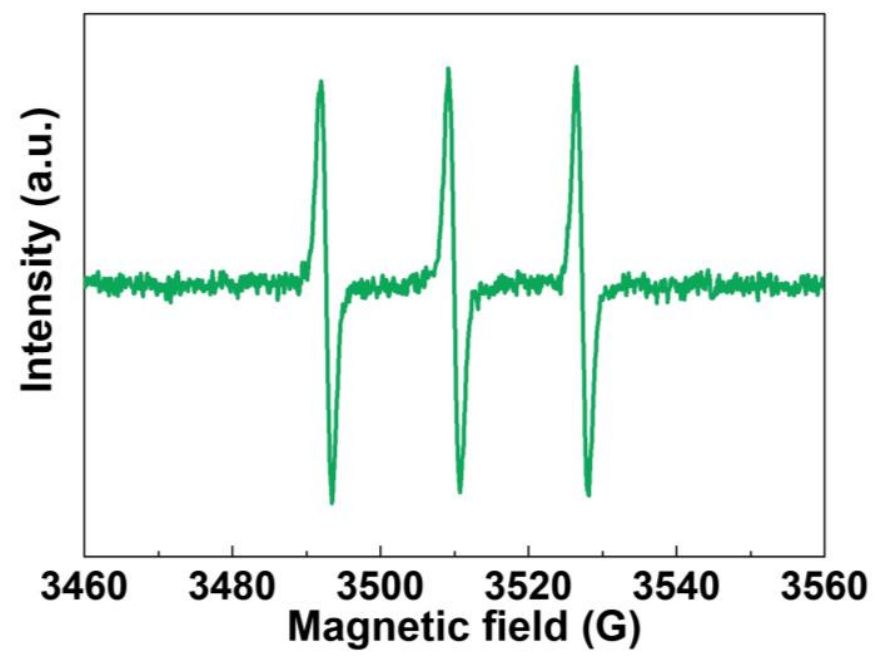

Figure S12. EPR spectrum of PMS activation with 50mM TEMP as spin trapping agent. 


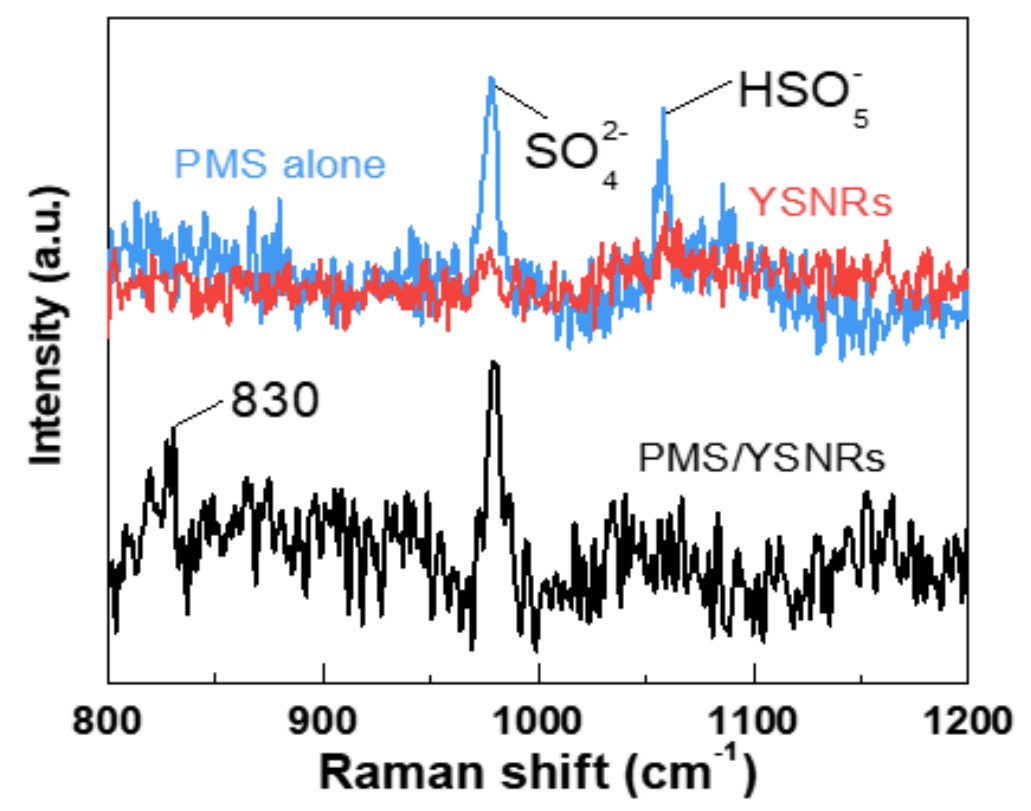

Figure S13. In-situ Raman spectra of PMS, YSNRs, and YSNRs/PMS. 


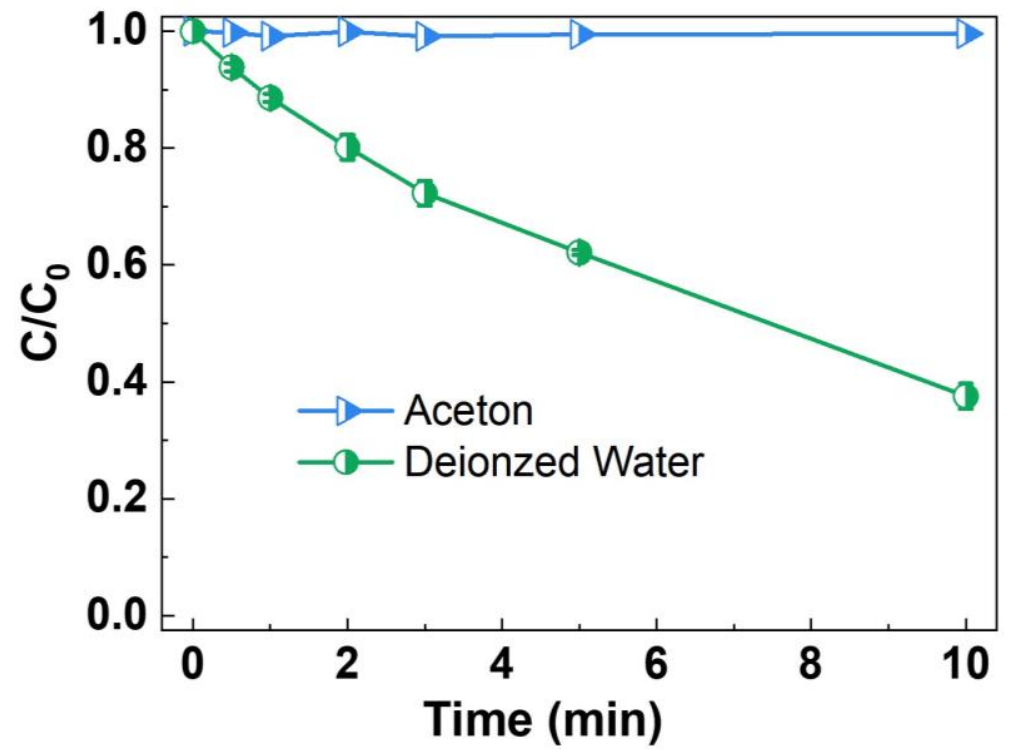

Figure S14. Removal efficiency of BPA with carbon-free catalysts. 
(a)

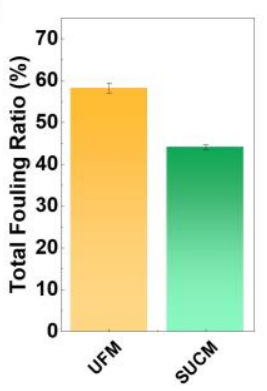

(b)

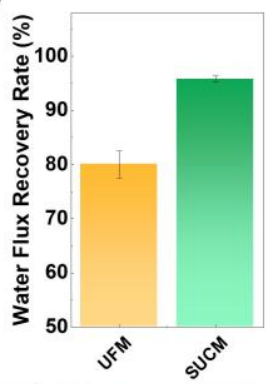

(c)

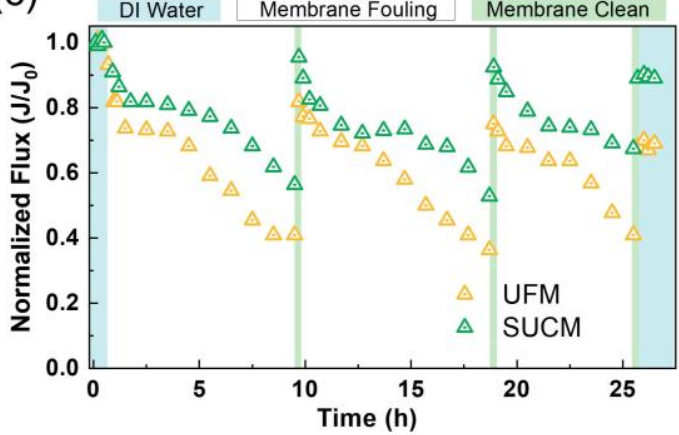

Figure S15. (a) Total fouling ratio and (b) water flux recovery rates of membranes after HA fouling and membrane cleaning. (c) Filtration cycles with multiple water flux recoveries for the SUCM and UFM. 


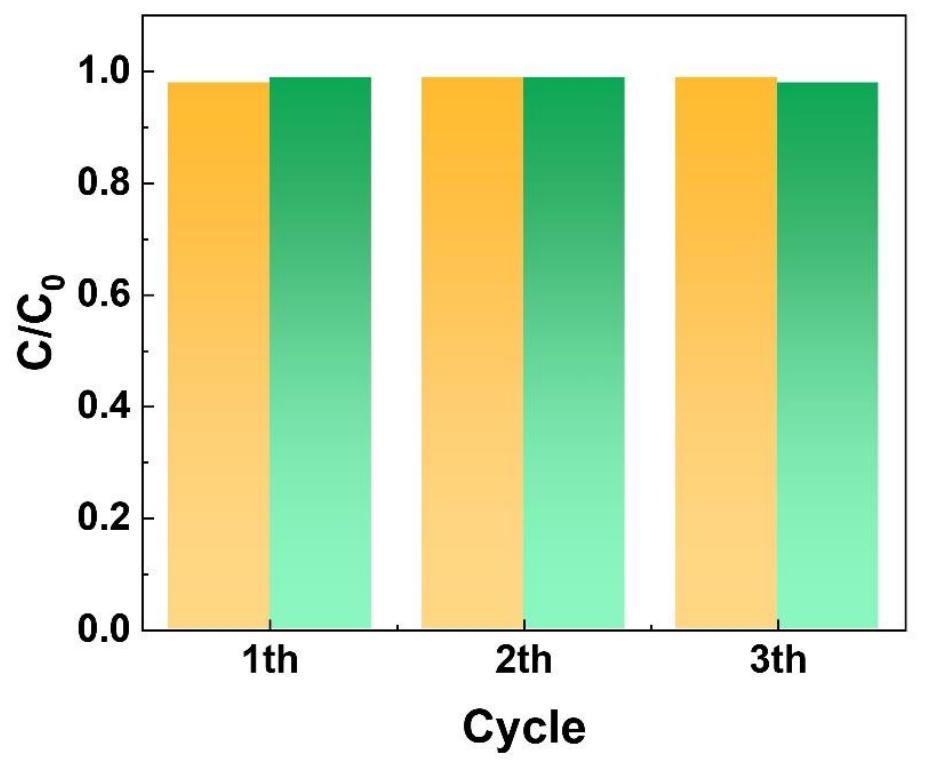

Figure S16. Normalized BPA concentration in the permeate solution of SUCM (green bar) and UFM (yellow bar). Samples were taken at the end of each filtration cycles of membrane fouling and cleaning experiments. 


\section{Reference}

1. Yang, D.; Feng, J.; Jiang, L.; Wu, X.; Sheng, L.; Jiang, Y.; Wei, T.; Fan, Z., Photocatalyst Interface Engineering: Spatially Confined Growth of ZnFe2O4 within Graphene Networks as Excellent Visible-LightDriven Photocatalysts. Adv Funct Mater 2015, 25, (45), 7080-7087.

2. Dotzauer, D. M.; Dai, J. H.; Sun, L.; Bruening, M. L., Catalytic membranes prepared using layer-by-layer adsorption of polyelectrolyte/metal nanoparticle films in porous supports. Nano Lett 2006, 6, (10), 2268-2272.

3. Immelman, E.; Bezuidenhout, D.; Sanderson, R. D.; Jacobs, E. P.; van Reenen, A. J., Poly(vinyl alcohol) gel sub-layers for reverse osmosis membranes. III. Insolubilization by crosslinking with potassium peroxydisulphate. Desalination 1993, 94, (2), 115-132. 